\title{
Influences of harvester and weather conditions on field loss and milling quality of rough rice
}

\author{
Ragab Khir ${ }^{1,2}$, Griffiths Atungulu ${ }^{3}$, Ding Chao ${ }^{4}$, Zhongli Pan ${ }^{1,5^{*}}$ \\ (1. Department of Biological and Agricultural Engineering, University of California, Davis, CA 95616, USA; \\ 2. Department of Agricultural Engineering, Faculty of Agriculture, Suez Canal University, Ismailia, Egypt; \\ 3. Department of Food Science, University of Arkansas, Fayetteville, AR 72701, USA; \\ 4. College of Food Science and Engineering, Nanjing University of Finance and Economies, Nanjing, Jiangsu 210023, China; \\ 5. Healthy Processed Foods Research Unit, Western Regional Research Center, Agricultural Research Service, \\ U.S. Department of Agriculture, Albany, CA 94710, USA)
}

\begin{abstract}
A better understanding of factors affecting field losses and milling quality during harvest is needed to improve the economic value of rice. The main objective of this study was to evaluate the impact of harvester header, harvester type, and weather conditions on field loss and milling quality of rough rice. Four popular harvesters equipped with different headers: John Deere 9870 with header D60-S, John Deere 9860 with header D972-S, Hardy, and John Deere 9770 with stripper header were evaluated in this study. The header and total field losses of rice were evaluated for each harvester under regular, rainy and windy weather conditions. Tests were conducted in a rice field cultivated with medium grain rice, variety M206, at Grimes, CA. Total rice yield (TRY), head rice yield (HRY) and whiteness index (WI) were examined to evaluate the effect of weather conditions on milling quality. The obtained results revealed that the rice field loss was affected by harvester header and type, and weather conditions. Among the conventional headers, D60-S significantly reduced the rice loss compared to the D972-S and the Hardy. Harvesting with John Deere 9860 resulted in significant header and field loss compared to other tested harvesters. The average loss increased from $236.2 \mathrm{~kg} / \mathrm{hm}^{2}$ and $445.2 \mathrm{~kg} / \mathrm{hm}^{2}$ under regular condition to $711.1 \mathrm{~kg} / \mathrm{hm}$ and $907.9 \mathrm{~kg} / \mathrm{hm}^{2}$ under windy condition for John Deere 9870 and John Deere 9860, respectively. The corresponding loss percentages increased from $2.6 \%$ and $4.9 \%$ to $7.9 \%$ and $10.1 \%$. HRY was significantly affected by weather condition at harvest time. However, the weather condition had no significant effect on TRY and WI. To minimize the rice field loss and obtain good milling quality, commercial rice harvesting during regular weather conditions is important. A proper combination of harvester type and header could be vital for reducing the rice loss.
\end{abstract}

Keywords: rice, harvesters, field loss, weather conditions, milling quality, harvester header

DOI: $10.25165 /$ j.ijabe.20171004.2993

Citation: Khir R, Atungulu G, Ding C, Pan Z L. Influences of harvester and weather conditions on field loss and milling quality of rough rice. Int J Agric \& Biol Eng, 2017; 10(4): 216-223.

\section{Introduction}

Rice loss in the field during harvesting presents a

\section{Received date: 2016-11-13 Accepted date: 2017-04-20}

Biographies: Ragab Khir, Postdoc Researcher, research interest: food engineering, Email: ragabkhir@yahoo.com; Griffiths Atungulu, Professor, research interest: food engineering, Email: atungulu@uark.edu; Ding Chao, Assistant Professor, research interest: food engineering, Email: kevindc1985@163.com.

*Corresponding author: Zhongli Pan, Research Engineer, research interest: food engineering. Healthy Processed Foods Research Unit, USDA-ARS Western Regional Research Center, 800 Buchanan St., Albany, CA 94710, USA. Tel: +1-510-5595861, Fax: +1-510-559-5851; Email: Zhongli.Pan@ars.usda.gov; zlpan@ucdavis.edu. direct economic loss to growers. In California, most rice growing areas encounter strong wind and rainfall events of various magnitudes during harvesting season, which cause rice lodging and moisture change and affect performance of harvesters, rice loss and milling quality $^{[1,2]}$. Also, in light of improved combine harvesters and emergence of new rice varieties, there is a need for continuous assessments of rice loss and milling quality $^{[3]}$. Understanding the impact of weather conditions, harvester headers, and harvester types on rice loss in the field and milling quality is of a great importance for mitigating rice loss and milling quality. 
To maximize profit in the rice harvesting process, an acceptable compromise must be sought between loss of grain yield, reduction in quality and maximizing harvest capacity. The loss of grain in the field caused by harvesters is classified into header loss (losses at the front of a harvester) and combining losses (losses at threshing, separation and cleaning units) or gathering loss and processing loss ${ }^{[4]}$. Header losses are known to vary widely with the type, variety and condition of the crop, and the designing features of the header ${ }^{[1]}$. The performance of modern rice harvesters, facilitated with conventional and stripper headers, is affected by the harvester settings and crop conditions ${ }^{[2-5]}$. Most harvester manuals give initial settings for the crop, however, operators must be prepared to adjust these based on conditions in a given field and the threshing characteristics of each rice variety. Harvesters must be adjusted to unique setting for each crop, variety, and maturity. Despite recommended setting, grain loss may be significant at the beginning and periodically throughout the harvest season ${ }^{[2]}$. In order to minimize such loss, a precise and accurate estimate is needed to make a suitable plan for an entire harvesting season.

The weather conditions that prevailed during the harvesting season have an impact on the grain loss and quality. These conditions are not controllable but should be observed because the harvesting schedule may have to be adjusted to minimize the field loss and quality degradation. Heavy rainfall during harvest delays harvesting which in effect increases both the yield and quality losses ${ }^{[6]}$. Moreover, the inherent characteristics of different varieties of rice, including shattering, lodging, and moisture absorption and desorption are affected by weather conditions ${ }^{[4]}$. Therefore, the suitable combination of harvesters used, crop conditions and weather conditions need to be selected to reduce harvest losses and improve produce quality ${ }^{[7]}$.

While grain loss is considered an important attribute when harvesting rice, the quality of rice is also a major concern. The quality of rice is determined mainly by head rice yield (HRY) and the presence of fines and foreign materials (dockage) ${ }^{[8]}$. HRY is the weight percentage of rough rice that remains greater than $75 \%$ of intact length throughout the milling process ${ }^{[3-9,10]}$. Improving HRY is a continuously targeted goal for rice growers. Weather conditions affect the moisture content of the rough rice at harvest thereby influencing HRY. Thompson and Mutters ${ }^{[1]}$ studied the effect of weather and rice moisture at harvest on milling quality of California medium-grain rice. They found that harvesting under dry climate conditions produced a wide range of head rice quality. Dry windy conditions during harvest caused rapid reductions in rice moisture, predisposing rice to significant HRY loss and most of the loss occurred after rehydration conditions. Dilday ${ }^{[12]}$ also stated that HRY generally decreased significantly as grain $\mathrm{MC}$ at harvest decreased. Moreover, Thompson and Mutters ${ }^{[1]}$ concluded that harvesting rice under a combination of dry meteorological conditions and conditions of diurnal rehydration is considered as a main reason for the commercial variation in California medium-grain rice quality. To produce the highest quantity and quality of rice possible, the effects of the combine header and type over the entire range of rice harvesting conditions need to be investigated.

Our previous study ${ }^{[13]}$ revealed that the loss in weight due to the presence of dockage was affected by the harvester header and type. The lowest average dockage occurred in the case of harvesters equipped with stripper headers compared to conventional headers. \#However, no significant difference was observed between dockage of rice harvested with John Deere with stripper header and that harvested with Claas Lexion with conventional header. It was also clear that besides the impact of harvester model, dockage was also significantly different within different header configurations. \#Additionally,\# rice dockage was affected by strong wind and rainfall events. Dockage was higher on windy and rainy days compared to during normal weather conditions. It was noted that fluctuations of rice moisture occurred due to strong winds and rains. It was also observed that strong wind caused rice lodging. Thus the harvesting operation may be negatively influenced. Particularly, rice cutting and feeding efficienciesłare compromised when rice stalk 
is fallen down. Consequently, this phenomenon may affect the grain losses from harvester header, threshing, separation and cleaning units as well.

Our elaborate review of previous studies demonstrated that there have been extensive combine tests performed on other grains, however, no research has been conducted in measuring rice harvesting losses induced by harvester header and type under various weather conditions. Moreover, little research has been conducted on the effect of weather conditions on rice milling quality ${ }^{[11,12,14,15]}$. Therefore, the objectives of this study were to: (1) determine the impact of harvester header and harvester type on rice losses in the field, (2) delineate effect of weather conditions on rice loss and rice milling quality, and (3) provide suitable recommendation for minimizing rice losses in the field and improving milling quality.

\section{Materials and methods}

\subsection{Harvesters}

Working with rice growers and the T\&P farms in California (CA), four popular rice harvesters were identified in order to investigate the impact of harvester type and header on the field loss. The tested harvesters were John Deere 9870 equipped with conventional header D60-S (Figure 1), John Deere 9860 with conventional header D972-S (Figure 2), Hardy with conventional header (Figure 3), and John Deere 9770 with stripper header (Figure 4). Configurations of harvester types and headers are presented in details in Table 1. Harvester tests were conducted in a rice field cultivated with medium grain rice, variety M206, at Grimes, CA. The rice loss was evaluated for each harvester under regular, rainy and windy weather conditions in the 2012 rice harvesting season. Based on harvester availability, the loss for the John Deere 9770 with stripper header was evaluated only under windy weather conditions. The regular weather condition means no rain or no strong winds. The operating parameters of the harvesters including forward speed, cylinder speed and cylinder clearance were maintained constant at conditions for optimal rice harvesting as recommended and used by the harvester operators.

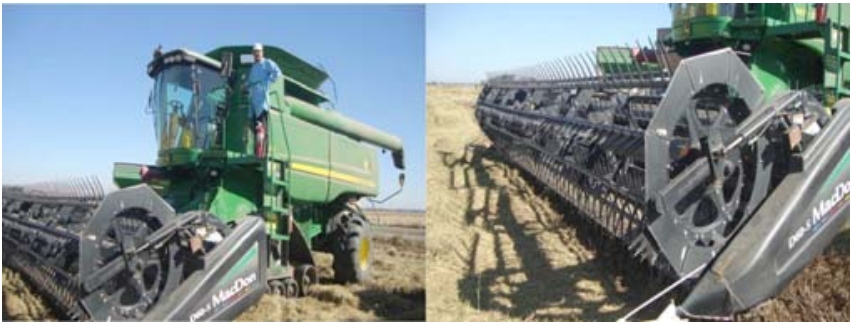

Figure 1 John Deer model 9870 with conventional header D60-S

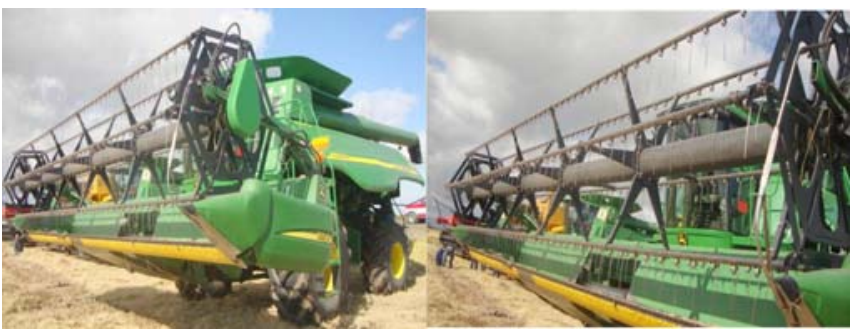

Figure 2 John Deer model 9860 with conventional header D972-S

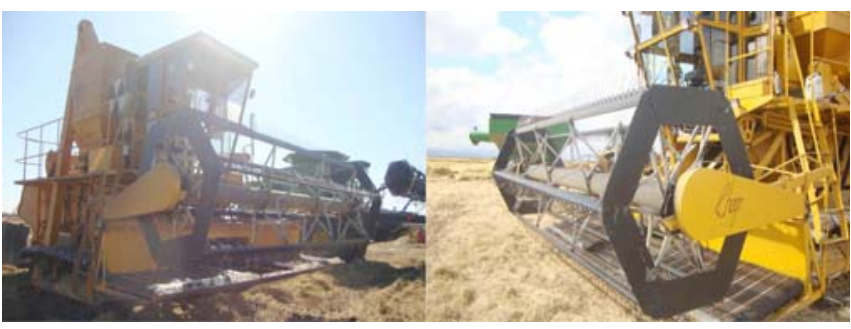

Figure 3 Hardy harvester with conventional header

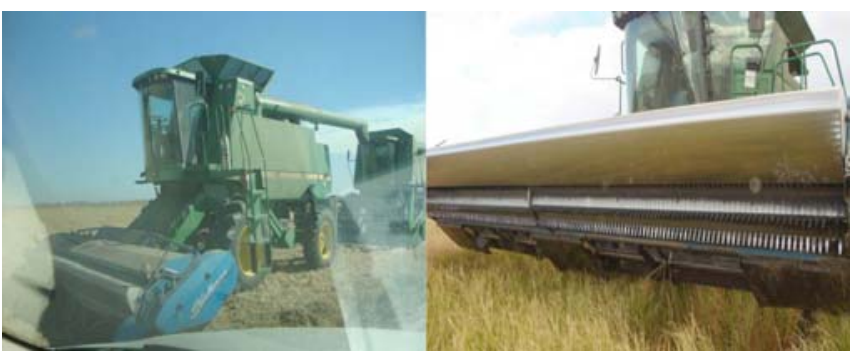

Figure 4 John Deer model 9770 with stripper header

Table 1 Configurations of tested harvester types and headers

\begin{tabular}{cccccc}
\hline \multirow{2}{*}{ Harvester } & Model & Year & \multicolumn{2}{c}{ Header } & \multirow{2}{*}{ Width $/ \mathrm{m}$} \\
\cline { 4 - 5 } & & & Type & Model & \\
\hline John Deere & 9870 & 2009 & Conventional & D60-S & 7.62 \\
John Deere & 9860 & 1997 & Conventional & D972-S & 7.62 \\
Hardy & Regular & 1980 & Conventional & Hardy & 5.48 \\
John Deere & 9770 & 2001 & Stripper & 9770 & 7.62 \\
\hline
\end{tabular}

\subsection{Evaluation of rice losses in the field}

Field loss of rice was considered to be the sum of header losses and losses after threshing, separating and cleaning by the combine. These two categories of losses were observed directly and studied separately. Field losses were determined for different harvesters under each tested weather conditions, including regular (John Deere 9870, Johon Deere 9860 and Hardy), rainfall (John Deere 870, John Deere 9860 and Hardy) and wind (John 
Deere 9870, John Deere 9860 and John Deere 9770). Weather conditions during the rice harvesting season were monitored to determine specific days with notable magnitudes of rainfall and strong winds. The conditions of tested weather events are described in details in Table 2. On each test day, the loss measurement evaluation commenced one hour after the beginning of harvesting operation. This allowed the tested harvesters to reach steady state operation. After steady state conditions were reached, six samples with three replicates were taken for each harvester for each test. Samples were taken from an area of one square foot $\left(0.09 \mathrm{~m}^{2}\right)^{[2-4,16,17]}$. Then the loss was calculated in germs per square meter. Three samples for header loss (HL) analysis were taken randomly from three locations under the harvester header. Another three samples for the threshing, separating and cleaning related losses (TSCLP) were taken from three locations at the rear of the harvester over $15 \mathrm{~m}$ of harvester travel ${ }^{[18]}$. The percentage of rice loss at the header and that due to threshing, separating and cleaning for each tested harvester was determined by Equations (1) and (2).

$$
H L P=\frac{H L}{H L+T S C L} \times 100
$$

where, $H L P$ is header loss percentage, $\%$; $H L$ is header loss, $\mathrm{g} / \mathrm{m}^{2} ;$ TSCL is threshing, separating and cleaning loss, $\mathrm{g} / \mathrm{m}^{2}$.

$$
T S C L P=\frac{T S C L}{T S C L+H L} \times 100
$$

where, TSCLP is threshing, separating and cleaning loss percentage.

\section{Table 2 Tested weather conditions}

\begin{tabular}{ccccc}
\hline Conditions & $\begin{array}{c}\text { Temperature } \\
/{ }^{\circ} \mathrm{C}\end{array}$ & $\begin{array}{c}\text { Relative humidity } \\
/ \%\end{array}$ & $\begin{array}{c}\text { Wind speed } \\
/ \mathrm{mph}\end{array}$ & $\begin{array}{c}\text { Precipitation } \\
\text { /in }\end{array}$ \\
\hline Regular & 29 & 57 & 2 & 0 \\
Rainfall & 17.8 & 83 & 6 & 1.3 \\
Wind & 14.5 & 67 & 15 & 0 \\
\hline
\end{tabular}

Rice harvesting under rainfall conditions commenced $12 \mathrm{~h}$ after the rainfall event. The losses were measured in grams per square meters and converted to kilograms per hectare. The total loss percentage was calculated by Equation (3):

$$
\operatorname{TYL}(\%)=\frac{F L}{T Y} \times 100
$$

where, TYL is total yield loss $\left(\mathrm{kg} / \mathrm{hm}^{2}\right) ; F L$ is field loss, and $T Y$ is total yield $\left(\mathrm{kg} / \mathrm{hm}^{2}\right)$ based on the weight of the crop production per hectare.

\subsection{Milling quality evaluation}

Milling quality evaluation was conducted for the rough rice samples collected at each weather condition. The initial moisture content (MC) of samples was $24.1 \% \pm 0.4 \%, 24.9 \% \pm 0.5 \%$ and $21.6 \% \pm 0.5 \%$ at regular, rainy and windy weather conditions, respectively. All reported MCs are averages of three replicates on wet basis and were determined by the air oven methods $\left(130^{\circ} \mathrm{C} \text { for } 24 \mathrm{~h}\right)^{[19]}$. To evaluate milling quality, the samples were dried from their initial moisture contents to moisture content of $13.0 \% \pm 0.4 \%$ using multiple drying passes of hot air at temperature of $43^{\circ} \mathrm{C}$ and velocity of $0.1 \mathrm{~m} / \mathrm{s}$. The drying conditions were similar to commercial drying practice. During each drying pass, the samples were heated for $20 \mathrm{~min}$ followed by tempering treatment for $4 \mathrm{~h}$ at ambient temperature. After drying, the rice samples were stored in zip-lock bags at room temperature of $(23.0 \pm 2.0)^{\circ} \mathrm{C}$ for one week before analyzing the milling quality. To conduct milling quality analysis, the rice samples (400 g each) were dehulled and milled using a Yamamoto Husker (FC-2K) and Yamamto Rice Mill (VP-222N, Yamamoto Co. Ltd., Japan). The samples were milled three times to achieve well-milled rice as defined by the Federal Grain Inspection Service (FGIS). The setting of throughput and whitening were 1 and 4 during the first two milling passes and 1 and 5 during the third milling pass. Total rice yield (TRY), head rice yield (HRY) and whiteness index (WI) were used to evaluate the effects of weather conditions on milling quality. The HRY was determined with Graincheck (Foss North America, Eden Prairie, MN). The WI was determined by the whiteness tester (C-300, Kett Electronic Laboratory, Tokyo, Japan). A higher index number indicates whiter milled rice. All reported milling quality indicators are averages of three replicates.

\subsection{Statistical analysis}

Data were analyzed statistically with SigmaStat (version 2.0, Jandel Corp., San Rafael, California) using one-way RM ANOVA and multiple comparisons. 
Tukey's adjustment method was used to compare means of rice loss and milling quality under different harvester headers, harvester types and weather conditions. Significance level was reported at $p<0.05$ level for all data.

\section{Results and discussion}

\subsection{Impact of harvester header on rice loss}

The average values and standard deviations of rice grain loss for different harvester headers under different weather conditions are presented in Table 3.

Table 3 Average header loss values and standard deviations for different headers

\begin{tabular}{cccc}
\hline Header type & Header model & Weather condition & Loss $/ \mathrm{g} \cdot \mathrm{m}^{-2}$ \\
\hline & \multirow{2}{*}{ D60-S } & Regular & $1.51 \pm 0.55^{\mathrm{a}}$ \\
& & Rainfall & $5.81 \pm 1.03^{\mathrm{b}}$ \\
\cline { 2 - 4 } Conventional & Wind & $2.70 \pm 0.34^{\mathrm{a}}$ \\
\cline { 2 - 4 } & D972-S & Regular & $9.47 \pm 1.23^{\mathrm{c}}$ \\
& & Rainfall & $23.14 \pm 3.18^{\mathrm{e}}$ \\
& & Wind & $5.81 \pm 0.97^{\mathrm{b}}$ \\
\hline Stripper & Hardy & Regular & $11.84 \pm 2.98^{\mathrm{d}}$ \\
& & Rainfall & $13.78 \pm 2.88^{\mathrm{d}}$ \\
\hline
\end{tabular}

Note: Superscript letters indicated that means with same letters designation in each column are not significantly different at $p<0.05$.

It can be seen that the rice loss was significantly $(p<0.05)$ affected by harvester header. In general, the three conventional headers had rice loss less than that of the single stripper header. Additionally, among the conventional headers, D60-S had significantly header loss compared to D972-S and Hardy. For example, the averages of rice loss under wind weather conditions were $(2.70 \pm 0.34) \mathrm{g} / \mathrm{m}^{2}$ and $(5.81 \pm 0.97) \mathrm{g} / \mathrm{m}^{2}$ for conventional headers (D60-S and D972-S) and $(18.41 \pm 1.51) \mathrm{g} / \mathrm{m}^{2}$ for the stripper header tested. The corresponding values of header loss percentage (HLP) were 3.8\%, 6.4\% and 32.3\%. The obtained results revealed that harvesting with D60-S significantly reduced rice header loss compared to the other harvesters tested. The stripper header had higher rice loss compared to the conventional headers tested. This finding is in agreement with that reported by Kalsirisilp and Singh $^{[20]}$. This may be due to the unacceptable shatter losses caused by the stripping elements $^{[5-21]}$. Additionally, strong winds results in rice lodging which causes incomplete detachment of plant parts for a subsequent threshing operation. Due to the harvesting schedule and the availability of the headers, the stripper header was tested only under the wind condition. To justify the stripper header loss, a further study is needed to evaluate the stripper header under regular and rain weather conditions.

\subsection{Impact of harvester type on rice loss}

The harvester type significantly affected the rice field loss. The John Deere 9870, John Deere 9770 and Hardy had much lower field loss compared to John Deere 9860. The averages of field loss were $(23.68 \pm 4.41) \mathrm{g} / \mathrm{m}^{2}$, $(20.02 \pm 3.01) \mathrm{g} / \mathrm{m}^{2}$, and $(44.56 \pm 5.56) \mathrm{g} / \mathrm{m}^{2}$ under regular condition and $(11.94 \pm 2.74) \mathrm{g} / \mathrm{m}^{2},(18.73 \pm 3.22) \mathrm{g} / \mathrm{m}^{2}$, and $(43.27 \pm 7.66) \mathrm{g} / \mathrm{m}^{2}$ under rainfall condition for John Deere 9870, Hardy and John Deere 9860, respectively (Table 4). The corresponding loss percentages were $2.36 \%, 2.00 \%$ and $4.45 \%$ under regular condition and $1.19 \%, 1.87 \%$ and $4.32 \%$ under rainfall condition. For wind condition, the averages of field loss were $(57.05 \pm 7.62) \mathrm{g} / \mathrm{m}^{2}$, $(71.15 \pm 6.89) \mathrm{g} / \mathrm{m}^{2}$ and $(90.10 \pm 7.86) \mathrm{g} / \mathrm{m}^{2}$ for John Deere 9770, John Deere 9870 and John Deere 9860, respectively (Table 4). The corresponding loss percentages were $5.70 \%, 7.11 \%$ and $9.10 \%$. It is important to notice that the increased rice loss for John Deere 9860 may be due to the increased loss at the threshing, separating and cleaning units of this harvester compared to the other tested harvesters. The threshing, separating and cleaning loss percentage (TSCLP) for John Deere 9860 was 78.7\%, 47.0\% and 93.0\% under regular, rainfall and wind conditions, respectively. While the TSCLP for hardy was $40 \%$ and $26 \%$ under regular and rainfall conditions, respectively.

The obtained results clearly indicated that harvesting using John Deere 9860 resulted in significant header and field losses compared to other tested harvesters. Additionally, the performance of the tested harvesters was affected by weather conditions during harvesting operation. For all tested harvesters, the losses from threshing, separating and cleaning units were significantly higher under wind condition compared to those under regular and rain conditions (Table 4). The impact of weather conditions on rice loss is discussed in details in the next section. 
Table 4 Average values and standard deviations of rice loss of tested harvesters under different weather conditions

\begin{tabular}{|c|c|c|c|c|c|}
\hline \multirow{3}{*}{ Harvester type } & \multirow{3}{*}{ Weather condition } & \multicolumn{4}{|c|}{ Loss } \\
\hline & & \multicolumn{2}{|c|}{ Threshing, separating and cleaning } & \multicolumn{2}{|c|}{ Field } \\
\hline & & $\mathrm{g} \cdot \mathrm{m}^{-2}$ & $\%$ & $\mathrm{~g} \cdot \mathrm{m}^{-2}$ & $\%$ \\
\hline John Deere (9870) with D60-S & & $22.06 \pm 3.86^{\mathrm{b}}$ & 2.20 & $23.68 \pm 4.41^{b}$ & 2.36 \\
\hline John Deere (9860) with D972-S & Regular & $35.09 \pm 5.72^{\mathrm{c}}$ & 3.50 & $44.56 \pm 5.56^{\mathrm{c}}$ & 4.45 \\
\hline Hardy & & $8.18 \pm 2.89^{\mathrm{a}}$ & 0.81 & $20.02 \pm 3.01^{\mathrm{b}}$ & 2.00 \\
\hline John Deere (9860) with D972-S & Rainfall & $20.12 \pm 2.82^{b}$ & 2.01 & $43.27 \pm 7.66^{\mathrm{c}}$ & 4.32 \\
\hline Hardy & & $4.95 \pm 0.65^{\mathrm{a}}$ & 0.49 & $18.73 \pm 3.22^{b}$ & 1.87 \\
\hline John Deere (9870) with D60-S & & $68.48 \pm 9.87^{\mathrm{d}}$ & 6.84 & $71.15 \pm 6.89^{\mathrm{e}}$ & 7.11 \\
\hline John Deere (9860) with D972-S & Wind & $85.03 \pm 6.78^{\mathrm{e}}$ & 8.50 & $90.10 \pm 7.86^{\mathrm{f}}$ & 9.10 \\
\hline John Deere (9770) with Stripper & & $38.64 \pm 4.22^{\mathrm{c}}$ & 3.86 & $57.05 \pm 7.62^{\mathrm{d}}$ & 5.70 \\
\hline
\end{tabular}

Note: Superscript letters indicated that means with same letters designation in each column are not significantly different at $p<0.05$.

\subsection{Impacts of weather conditions on rice loss}

Average rice grain losses for the tested harvesters under regular, rainfall and wind conditions are shown in Figure 5. The results revealed that the weather condition significantly affected the performance of tested harvesters. Particularly, the rice loss significantly increased during wind condition. The average loss increased from $236.2 \mathrm{~kg} / \mathrm{hm}^{2}$ and $445.2 \mathrm{~kg} / \mathrm{hm}^{2}$ under regular condition to $711.1 \mathrm{~kg} / \mathrm{hm}^{2}$ and $907.9 \mathrm{~kg} / \mathrm{hm}^{2}$ under wind condition for John Deere 9870 and John Deere 9860, respectively (Figure 5). The corresponding loss percentages increased from $2.6 \%$ and $4.9 \%$ to $7.9 \%$ and $10.1 \%$ when the rice yield was assumed as 8000 pound/acre $\left(8990 \mathrm{~kg} / \mathrm{hm}^{2}\right)$ (Figure 6). Additionally, the rice loss at rainfall condition was slightly less than that at regular condition. Increase in rice loss at wind condition may be due to the decreased moisture content and rice lodging compared to regular and rain conditions. The moisture content of harvested rice was $24.1 \% \pm 0.4 \%$, $24.9 \% \pm 0.5 \%$, and $21.6 \% \pm 0.5 \%$ at regular, rainfall and wind conditions, respectively. It is clearly seen that John Deere 9860 had the highest loss under all tested weather conditions (Figure 6). The increased rice loss for John Deere 9860 is related to increasingly integrated loss for both header and threshing, separating and cleaning units especially under the wind condition. Most losses were related to threshing, separating and cleaning rather than conventional headers on John Deere 9870 and Hardy.

The obtained results under tested conditions revealed that harvester headers and types affect rice field loss. At the same time, weather conditions affected the performance of all tested harvesters. Losses from threshing, separation and cleaning of rice from the combine were significantly higher under windy conditions, up to $10 \%$ compared to those under regular or rainy conditions. The use of conventional harvester header could cause less field loss compared to the single stripper header observed under wind weather conditions. Use of appropriate harvesters and header type with optimal settings and at the regular weather condition could improve the economic value of rice.

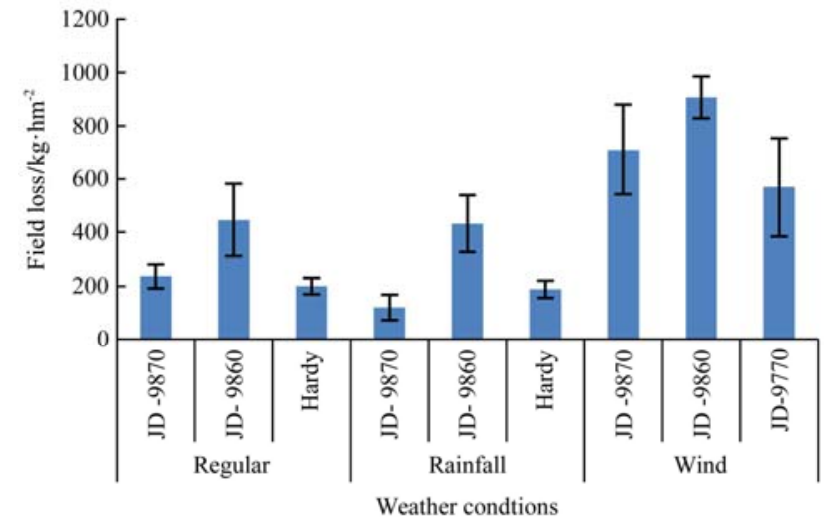

Figure 5 Rice field loss for different harvesters and weather conditions (JD: John Deere)

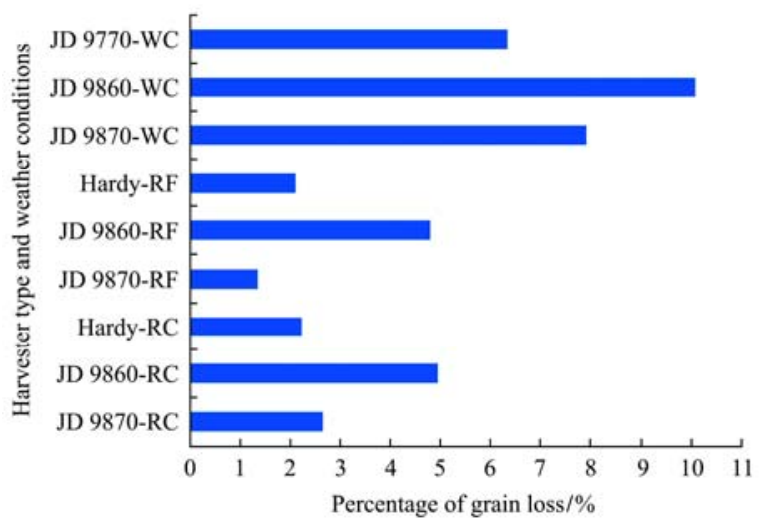

Figure 6 Percentage of rice loss for different harvesters and weather conditions. (JD: John Deere, RC: Regular condition, RF: Rainfall condition, WC: Wind condition) 


\subsection{Impacts of weather condition on rice milling} quality

The milling quality indicators, including TRY, HRY and WI for rough rice harvested under regular, rainfall and wind conditions are presented in Figures 7 and 8. The results indicated that the weather condition had no significant effect on TRY and WI. However, the weather condition had a significant effect on HRY (Figure 7). For example, the averages of TRY were $68.2 \%, 68 \%$ and $69.2 \%$ for regular, rainfall and wind conditions, respectively (Figure 7). The corresponding values of HRY were $60.8 \%, 51.4 \%$ and $60.3 \%$ (Figure 7). It can be seen that the rainfall condition significantly decreased HRY compared to regular and wind conditions. This indicated that fissured kernels increased and were primarily caused by rapid moisture adsorption due to rainfall and changes in humidity. These results are in agreement with those reported by Jodari and Lindscombe ${ }^{[22]}$. Where both long and medium grain rice kernels experienced a significant reduction in HRY under rain events. Finassi et al. ${ }^{[15]}$ found that dry conditions allowed $\mathrm{MC}$ to decrease and subsequent rain caused a significant drop in HRY for medium grain rice grown in Italy. Thompson and Mutters ${ }^{[11]}$ reported that dry windy conditions during harvest caused a significant HRY loss due to rapid reductions in rice moisture and subsequent rehydration conditions. Dilday ${ }^{[12]}$ stated that HRY generally decreased significantly as grain MC at harvest decreased. Some previous researches ${ }^{[14,23-25]}$ also indicated that cycles of drying and moisture absorption due to rain and humid weather conditions cause paddy rice kernels to fissure and negatively influences the milling quality.

When the results of WI were examined, there was no significant difference among the tested conditions (Figure 8). For instance, values of WI were 39.7, 40.4 and 39.2 units for regular, rainfall and wind conditions, respectively (Figure 8). The WI under rainfall condition was slightly higher than that of regular and wind conditions, but they were not significantly different. The obtained results revealed that weather condition has some impact on HRY. Accordingly, to minimize the rice field loss and obtain good milling quality, it is important to conduct commercial rice harvesting during regular weather conditions with selected combines.

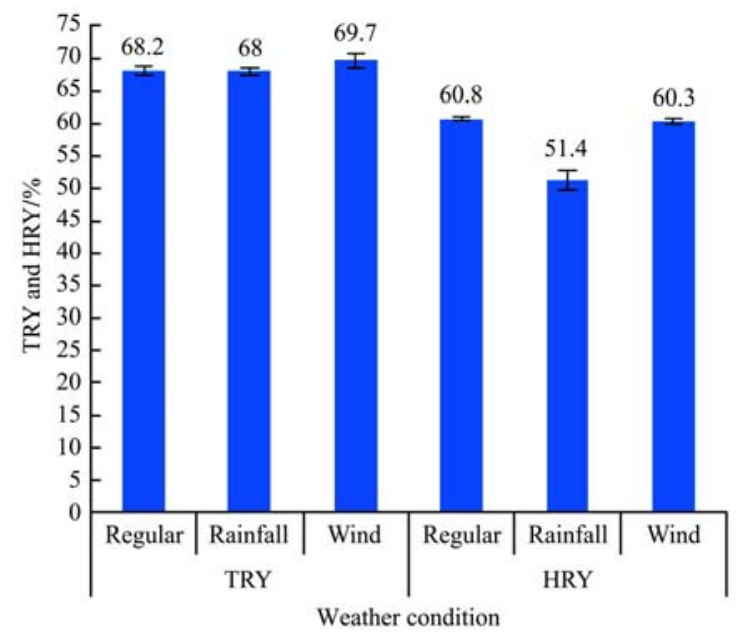

Figure 7 Total rice yield (TRY) and head rice yield (HRY) of rough rice (M206) harvested under different weather conditions

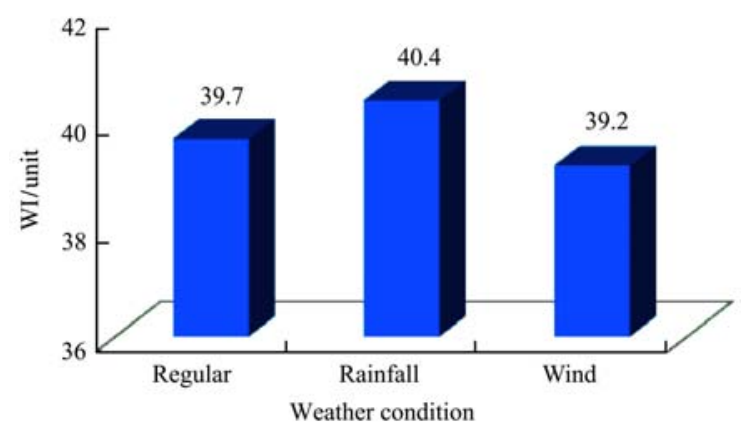

Figure 8 Whiteness index (WI) of rough rice (M206) harvested under different weather conditions

\section{Conclusions}

The research showed that the rice field loss and milling quality were affected by harvester headers, harvester types and weather conditions. Among the conventional headers, D60-S significantly reduced rice loss compared to the D972-S and the Hardy. Harvesting with the John Deere 9860 harvester resulted in significant header and field loss compared to the other harvesters tested. Additionally, weather conditions affected the performance of all tested harvesters. Losses for threshing, separating and cleaning of rice from the tested combines were significantly higher under windy condition which was up to $10 \%$ compared to those under regular and rainy conditions. The weather condition also affected milling quality, especially head rice yield (HRY). Rainy conditions significantly decreased HRY compared to regular and windy conditions. Weather condition had no significant effect on total rice yield (TRY) and 
whiteness index (WI). Thus, harvesting under regular weather conditions is recommended to minimize field loss and to maintain good milling quality. Use of a conventional header could cause less field loss than a stripper header under wind weather conditions. However, to determine the best combination of harvester type and header, it is vital to understand harvesterdependent losses from threshing, separation and cleaning within the combine.

\section{Acknowledgements}

The investigators would like to express their appreciation for the great support received from the following organization: USDA-ARS-WRRC, California Rice Research Board, and T\&P Farms in California.

\section{[References]}

[1] Klinner W E, Biggar G W. Some effects of harvest date and design features of the cutting table on the front losses of combine-harvesters. J. Agr. Eng. Res., 1972; 17: 71-78.

[2] Mutters R G, Thompson J F. Rice quality handbook. Publication 3514. Oakland, Cal. University of California, Department of Agricultural and Natural Resources, 2009.

[3] Andrews S B, Siebenmorgen T J, Vories E D, Loewer D H, Mauromoustakos A. Effects of combine operating parameters on harvest loss and quality in rice. Trans. ASAE, 1993; 36(6): 1599-1607.

[4] Dodds M E. Grain losses in the field when windrowing and combining barley. Canadian Agr. Eng., 1974; 16(1): 6-9.

[5] Klinner W E, Neale M A, Arnold R E, Geikie A A, Hobson R N. A new concept in combine harvester headers. J. Agr. Eng. Res., 1987; 38: 37-45.

[6] Nawi N M, Chen G, Zare D. The effect of different climatic conditions on wheat harvesting strategy and return. Bio. Eng., 2010; 106: 493-502.

[7] Adisa A F, Ndirika V I O, Yiljep Y D, Mohammed U S. Determination of optimum operational conditions of a grain stripper header for rice harvesting in Nigeria. Int. J. Eng. Tech., 2010; 2(7): 1292-1300.

[8] Andrews S B, Siebenmorgen T J, Loewer D H. Combine test system for determining harvest loss in rice. Appl. Eng. Agr., 1992; 8(6): 759-763.

[9] USDA-FGIS. Rice inspection handbook. Washington D C, USDA Agricultural Marketing Service, 1994.

[10] Bhattacharya K R. Analysis of rice quality. In rice quality:
A guide to rice properties and analysis, Cambridge, UK Woodhead Publishing, 2011; 431-530.

[11] Thompson J F, Mutters R G. Effect of weather and rice moisture at harvest on milling quality of California medium-grain rice. Trans. ASABE, 2006; 49(2): 435-440.

[12] Dilday R H. Milling quality of rice: Cylinder speed vs. grain-moisture content at harvest. Crop Sci., 1989; 29(6): $1532-1535$.

[13] Atungulu G G, Prakash B, Wang X, Wang T, Fu R, Khir R, et al. Determination of dockage for accurate rough rice quality assessment. Appl. Eng. Agr., 2013; 29(2): 253-261.

[14] Siebenmorgen T J, Perdon A A, Chen X, Mauromoustakos A. Relating rice milling quality changes during adsorption to individual kernel moisture content distribution. Cereal Chem., 1998; 75(1): 129-136.

[15] Finassi A, Norris P, Vidotto F. The effect of weather on whole-kernel milling yield of selected cultivars of Oryza sativa L. grown in the Po Vally of Italy. Manila, Philippines: International Rice Research Institute. In Proc. $2^{\text {nd }}$ Temperate Rice Conference, 2002; 471-479.

[16] Schuler R T, Rodakowski N N, Kucera H L. Grain harvesting losses in North Dakota. Farm research, 1975; 20-21.

[17] Mostofi S M R. Filed evaluation of grain loss monitoring of combine JD 955. Adv. Environ. Biol., 2010; 4(2): 162-167.

[18] ASAE Standards. S396.2: Combine capacity and performance test procedure. $45^{\text {th }}$ ed. St. Joseph, Mich.: ASAE, 1998.

[19] ASAE Standards. S352.2: Moisture measurementUngrounded grain and seeds. $42^{\text {nd }}$ ed. St. Joseph, Mich.: ASAE, 1995.

[20] Kalsirisilp R, Singh R. Adoption of a stripper header for a Thi-made rice combine harvester. J. Agr. Eng. Res., 2001; 80(2): 163-172.

[21] Tado C J M, Wacker P, Kutzbach D, Suministrado D C. Development of stripper harvester: A review. J. Agr. Eng. Res., 1998; 71(1): 103-112.

[22] Jodari F, Lindscombe S D. Grain fissuring and milling yields of rice cultivars as influenced by environmental conditions. Crop Sci., 1996; 36(6): 1496-1502.

[23] Kunze O R. Practical relative humidity systems that fissure rice and other grains. Appl. Eng. Agr., 1993; 9(1): 85-89.

[24] Geng S, Williams J F, Hill J E. Harvest moisture effects on rice milling quality. California Agr., 1984; 38(11/12): 11-12.

[25] Lan Y, Kunze O R, Lague C, Kocher M F. Mathematical model of the distribution of stress within a rice kernel from moisture adsorption. J. Agr. Eng. Res., 1999; 72(3): $247-257$. 